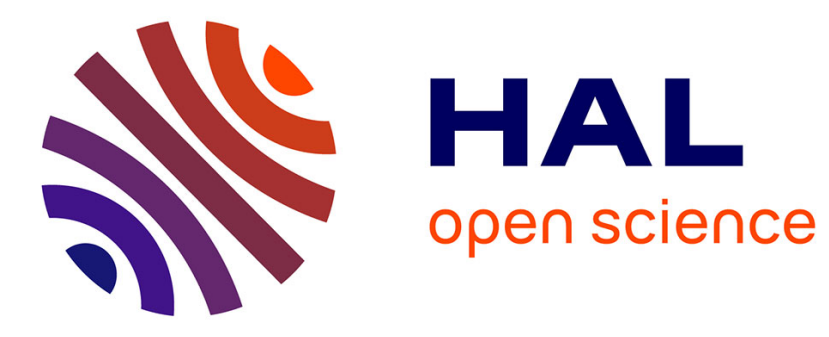

\title{
Production of lipophilic nanogels by spontaneous emulsification
}

Shukai Ding, Bilal Mustafa, Nicolas Anton, Christophe Serra, Delphine Chan-Seng, Thierry Vandamme

\section{- To cite this version:}

Shukai Ding, Bilal Mustafa, Nicolas Anton, Christophe Serra, Delphine Chan-Seng, et al.. Production of lipophilic nanogels by spontaneous emulsification. International Journal of Pharmaceutics, 2020, 585, pp.119481. 10.1016/j.ijpharm.2020.119481 . hal-02895292

\section{HAL Id: hal-02895292 \\ https://hal.science/hal-02895292}

Submitted on 9 Jul 2020

HAL is a multi-disciplinary open access archive for the deposit and dissemination of scientific research documents, whether they are published or not. The documents may come from teaching and research institutions in France or abroad, or from public or private research centers.
L'archive ouverte pluridisciplinaire HAL, est destinée au dépôt et à la diffusion de documents scientifiques de niveau recherche, publiés ou non, émanant des établissements d'enseignement et de recherche français ou étrangers, des laboratoires publics ou privés. 


\title{
Production of lipophilic nanogels by spontaneous emulsification
}

\author{
Shukai Ding, ${ }^{1,2, \#}$ Bilal Mustafa, ${ }^{1,3,4, \#}$ Nicolas Anton, ${ }^{3, *}$ Christophe A. Serra, ${ }^{1, *}$ Delphine \\ Chan-Seng, ${ }^{1}$ Thierry F. Vandamme ${ }^{3, *}$ \\ ${ }^{1}$ Université de Strasbourg, CNRS, Institut Charles Sadron UPR 22, F-67000 Strasbourg, France \\ ${ }^{2}$ Shaanxi University of Science \& Technology, Institute of Atomic and Molecular Science, CN-710021, Xi'an, Shaanxi, China \\ ${ }^{3}$ Université de Strasbourg, CNRS, CAMB UMR 7199, F-67000 Strasbourg, France \\ ${ }^{4}$ Faculty of Pharmacy, University of Sindh, Jamshoro, Sindh, Pakistan
}

* Corresponding authors: C.A. Serra, Tel: + 33388414 095, Email: ca.serra@unistra.fr, N. Anton, Tel: +33 368854 251, Email: nanton@unistra.fr, T. Vandamme, Tel: +33 368854 106, Email: vandamme@unistra.fr.

\# SD and BM have contributed equally 


\begin{abstract}
Nanosized gel particles, so-called nanogels, have attracted substantial interest in different application fields, thanks to their controllable and three-dimensional physical structure, good mechanical properties and potential biocompatibility. Literature reports many technologies for their preparation and design, however a recurrent limitation remains in their broad size distributions as well as in the poor size control. Therefore, the monodisperse and size-controlled nanogels preparation by simple process -like emulsification- is a real challenge still in abeyance to date. In this study we propose an original low energy emulsification approach for the production of monodisperse nanogels, for which the size can be finely controlled in the range 30 to $200 \mathrm{~nm}$. The principle lies in the fabrication of a direct nano-emulsion containing both oil (medium chain triglycerides) and a bi-functional acrylate monomer. The nanogels are thus formed in situ upon UV irradiation of the droplet suspension. Advantage of such modification of the oil nano-carriers are the potential modulation of the release of encapsulated drugs, as a function of the density and/or properties of the polymer chain network entrapped in the oil nano-droplets. This hypothesis was confirmed using a model of hydrophobic drug ketoprofen- entrapped into the nanogels particles, along with the study of the release profile, carried out in function of the nature of the monomers, density of polymer chains, and different formulation parameters.
\end{abstract}




\section{Introduction}

Nanogels can be defined as a three-dimensional cross-linked polymer network dispersed as nanoparticle and swollen by a liquid phase. Nanogels have receive considerable attention in many aspects due to their potential applications such as sensing (Peng et al., 2010), diagnostics (Oishi et al., 2007, Hasegawa et al., 2005), bioengineering (Hayashi et al., 2009) and drug delivery (Qiao et al., 2011). In contrast with conventional polymer nanoparticles which exhibit a packed matrix structure, nanogels exhibit a much higher loading capability, and can be considered as hybrid cargo between emulsions, capsules, and polymer nanoparticles. Molecules of interest can be trapped inside the gel swelling phase and, according to the properties of the polymer network their delivery can be tuned and finely controlled. Nanogels have been formulated by various strategies based on the gelation of polymers already solubilized in the dispersed phase, by either covalent or non-covalent cross-linking. The first approach covalent cross-linking results in the gelation of nano or macro-emulsion (McAllister et al., 2002), fabrication of polymerized block copolymer micelles (Chacko et al., 2012) or nano-template method in which the particle size in controlled by well-defined templates.(Sasaki and Akiyoshi, 2010, Yan et al., 2017). The polymer chemistry was carried out through different ways, such as cross-linking on disulfide groups (Ryu et al., 2010b) and amide cross-linking (Zhuang et al., 2012) similarly to those existing in biological environment. On the other hand, nanogels were also fabricated through polymer cross-linking by non-covalent interactions, such as hydrophobic interactions, hydrogen bonding, van der Waals interactions.(Raemdonck et al., 2009, Gruber et al., 2018, García and Cuggino, 2018). Importantly, most of the methods reported in literature deal with the fabrication of hydrogels or nano-hydrogels thus using watersoluble polymers (Ryu et al., 2010a, Torchilin, 2005). However, the encapsulation of hydrophobic and / or lipophilic molecules is only described in a few reports, in the case of micelle cross-linking (O'Reilly et al., 2006) or through self-assembly of amphiphilic polymers (Ryu et al., 2010b).

In this study, we developed a novel approach to formulate and synthetize lipophilic nanogels based on spontaneous emulsification of an \{oil + monomer\} phase, followed by in situ photopolymerization of the nano-droplets. The oil phase is composed of a mixture of a monofunctional monomers (ethyl acrylate, butyl acrylate), difunctional monomer (tri(propylene glycol) diacrylate, TPGDA), vegetable oil (medium chain triglycerides), and photoinitiators (detailed in the experimental section). The process was based on the fact that the 
monomeric phase behaves very similarly as vegetable oils, as regards the spontaneous emulsification process (Anton and Vandamme, 2011, Wang et al., 2019). The spontaneous process is indeed related to the respective affinities between the oil phase, the nonionic surfactant, and the aqueous phase; in this study, TPGDA shows a behavior close to the one of oils, that allows to obtain nanogels with a controllable size and homogeneous size distribution. Herein we investigated the nanogels formation, the impact of the chemical and formulation parameters on suspension properties, and, on the second hand, the release profiles of model drug encapsulated. Ketoprofen as model of hydrophobic drug nonsteroidal anti-inflammatory having analgesic and antipyretic properties used in the treatment of rheumatic and nonrheumatic inflammatory disorders (Molina-García et al., 2013, Khan et al., 2013) was encapsulated and its controlled release investigated.

\section{Experimental section}

\subsection{Materials}

Medium chain triglycerides (MCT, Labrafac ${ }^{\circledR}$ WL 1349) was purchased to Gattefossé S.A., Saint-Priest, France. Tri(propylene glycol) diacrylate (TPGDA), ethyl acrylate (EA), $n$-butyl acrylate (nBA), 1-hydroxycyclohexyl phenyl ketone (HCPK, as photoinitiator, 99\% purity) were obtained from Sigma-Aldrich. Water used was milliQ water, and a nonionic hydrophilic surfactant (Kolliphor ELP ${ }^{\circledR}$ ) was purchased from BASF, Ludwigshafen, Germany. It is a polyethoxylated-35 castor oil with a Hydrophilic-Lipophilic Balance (HLB) of $12 \sim 14$. Ketoprofen was obtained from S.I.M.S (Florence, Italy).

\subsection{Preparation of nanogels}

\subsubsection{Nano-emulsification}

The general principle of spontaneous nano-emulsification is the sudden mixing of an oil phase homogenized with hydrophilic surfactants, at elevated temperature with the aqueous phase at room temperature. As a result of the rapid change in the surfactant solubility between oil and water, the oil phase is broken-up and generate nano-emulsion. (Rehman et al., 2019) The water phase brutally integrates the oily one, fast enough to make the oil molecules to undergo a spinodal-like decomposition generating the oil droplets. (Miller, 1988) In this study, the oil phase was a mixture of MCT and TPGDA two components extremely miscible each other at different weight contents. The concentration of TPGDA in the oil phase was varied from 
$10 \mathrm{wt} . \%$ to $50 \mathrm{wt} . \%$. Then, the formulation parameter relating the mixing proportions with the nonionic surfactant was noted surfactant-to-oil weight ratio, defined as $\mathrm{SOR}=100 \times \mathrm{w}_{\text {surfactant }}$ $/\left(\mathrm{w}_{\text {surfactant }}+\mathrm{w}_{\mathrm{oil}}\right)$, where $\mathrm{w}$ indicates the weight of the different compounds, was varied from $20 \%$ to $70 \%$. Finally, the water content in all the formulations was kept constant at $60 \mathrm{wt} . \%$ of the final suspension. Once the $\{$ oil + surfactant $\}$ phase was mixed, homogenized (vortex), and set at $75^{\circ} \mathrm{C}$, the aqueous phase at room temperature was suddenly added in it and the whole mixture was again homogenized (vortex) for one minute, up to the nano-emulsions was completely generated and homogeneous. Variations of the monomer composition were also studied, substituting a part of TPGDA for either $n$-butyl acrylate or ethyl acrylate (details below).

\subsubsection{Polymerization in nano-droplets}

As illustrated in Figure 1 (a) stable nano-emulsions were generated by a simple phase mixing (see sub-section above) and vortex phase homogenization. The freshly prepared nanoemulsions were polymerized by UV irradiation in a tube-in-tube device (Figure 1 (b)). The nano-emulsions were charged in a $10 \mathrm{~mL}$ plastic syringe (HSW, Norm-Ject, France), placed in the syringe pump (KDS LEGATO 200, KdScientific) holder, delivering the nano-emulsion through a $30 \mathrm{~cm}$ long polytetrafluoroethylene (PTFE) tubing (1.68 mm ID x $3.2 \mathrm{~mm}$ OD) at a constant flowrate of $63 \mu \mathrm{L} / \mathrm{min}$. The PTFE tubing was inserted inside a $4 \mathrm{~cm}$ ID stainless steel tube equipped at both ends with a T-junction (Swagelok) in which were set the two light guides of an UV source (Lightningcure LC8, Hamamatsu) operating at $\lambda=365(\mathrm{~nm})$ wavelength corresponding to the maximum of absorbance of the photoinitiator (HCPK) added into the nano-emulsion`s monomer phase (3.5 wt.\% of monomer phase). According to the size of tubing and flow rate, the residence time of the nano-emulsions under the UV irradiation was about 11 min. The final resulting polymerized nano-emulsion, i.e. nanogel was collected as homogeneous and clear sample, visually similar to common nano-emulsions 

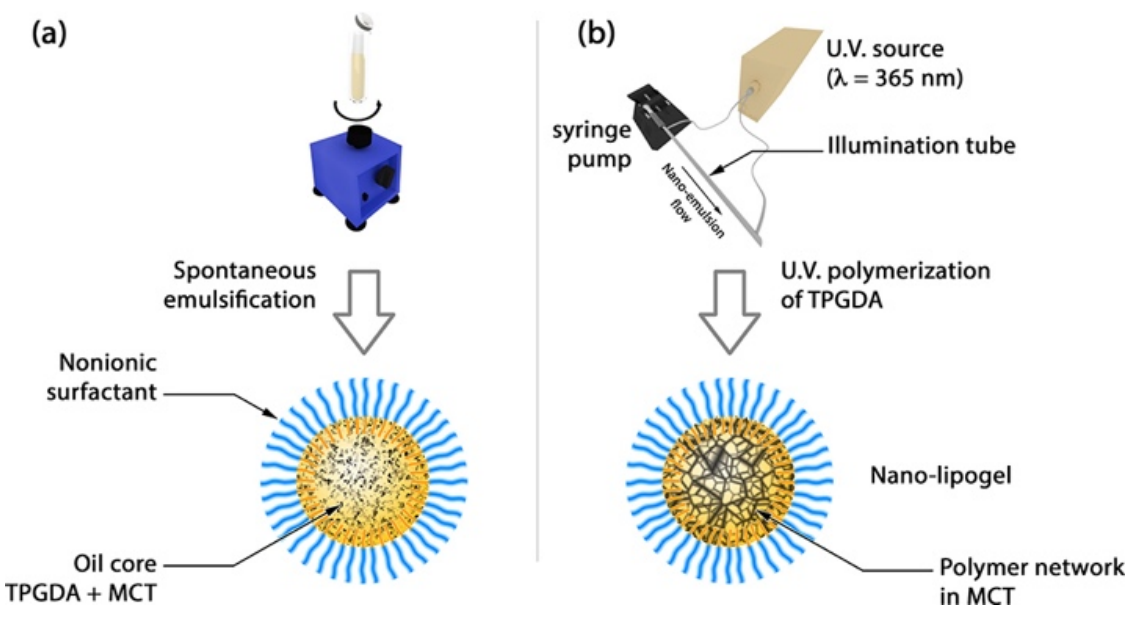

Figure 1: Schematic drawings of (a) the low-energy nano-emulsification of non-polymerized nano-droplets suspension, and (b) continuous-flow U.V. polymerization setup producing nanogels.

\subsubsection{Preparation of ketoprofen-loaded nanogels}

Ketoprofen-loaded nanogels containing a combination of difunctional and monofunctional monomers (TPGDA, EA and BA) were prepared by mixing the drug into the MCT (Labrafac ${ }^{\circledR}$ WL) at $0.5 \mathrm{wt} . \%$. While the impact of monomer concentration in oil monomer to oil weight ratio was varied and studied regarding the study of the formulation, in this stage of ketoprofen encapsulation and release, this ratio was chosen to be 50\%, and SOR at $40 \%$. The monomer phase was composed of pure TPGDA $(50 \%)$ or a mixture of TPGDA $(7.5 \mathrm{wt} \%)$ with either EA or BA (42.5 wt.\%).

\subsubsection{Dynamic light scattering}

Hydrodynamic diameter, the size distribution and polydispersity indexes (PDI) of the nanogels suspensions were assessed by dynamic light scattering (DLS) using a Malvern Nano ZS instrument (Malvern, Orsay, France). The helium-neon laser (4 $\mathrm{mW})$ was operated at $633 \mathrm{~nm}$, the scatter angle was fixed at $173^{\circ}$ and the sample temperature was maintained at $25^{\circ} \mathrm{C}$. The PDI of the particle size distribution is a measure of the broadness of the size distribution and it is commonly admitted that PDI values below 0.2 corresponds to monomodal distributions. DLS measurements were performed in triplicates after dilution (at 1/50) in ultrapure water.

\subsubsection{UV spectrophotometry for drug release measurement}

Ketoprofen release studies were carried out with a dialysis tubing (regenerated cellulose, MWCO 12-14000 Daltons, Medicell International Ltd, London, U.K.). Two grams of freshly 
prepared nanogel emulsions were charged into the dialysis tubing which was then immersed into $500 \mathrm{~mL}$ of Phosphate Buffer Solution USP $(6.8 \mathrm{pH})$ gently agitated by magnetic agitation at $250 \mathrm{rpm}$ and $(37 \pm 1){ }^{\circ} \mathrm{C}$. Samples of dialysate $(2 \mathrm{~mL})$ were collected after predetermined time in such a way that this amount of dialysate was replaced by same amount of medium prewarmed at $37^{\circ} \mathrm{C}$ to insure sink conditions. The concentration of ketoprofen in the dialysate was determined with UV spectrophotometer (Cary 4000-HP, Agilent technologies) operating at 260 $\mathrm{nm}$, using a calibration curve beforehand established (with linear extrapolation fitting with: Abs $=\mathrm{C} \times 0.0673+0.0011$, with $\mathrm{Abs}$ is the light absorbance and $\mathrm{C}$ the ketoprofen concentration in $\mathrm{ug} / \mathrm{ml}, \mathrm{R}^{2}=0.999$, data not shown). Experiments were performed in triplicate.

It is important to note here that the drug release through a dialysis bag, in some conditions, can be shifted from the actual drug release from the drug carrier. As reported in a recent study (Yu et al., 2019) the difference between the actual drug release from the nanocarrier and the one in the release medium outside of the dialysis bag can be impacted by both the cut-off and the nature of the membrane. However, in the present study, the experimental conditions used, $12 \mathrm{kDa}$ and $\mathrm{RC}$ membrane, fit with the ones for which the drug release outside of the dialysis bag are almost superimposed with the actual drug release from its carrier.

\section{Results and discussions}

\subsection{Impact of the formulation parameters on the nanogel size}




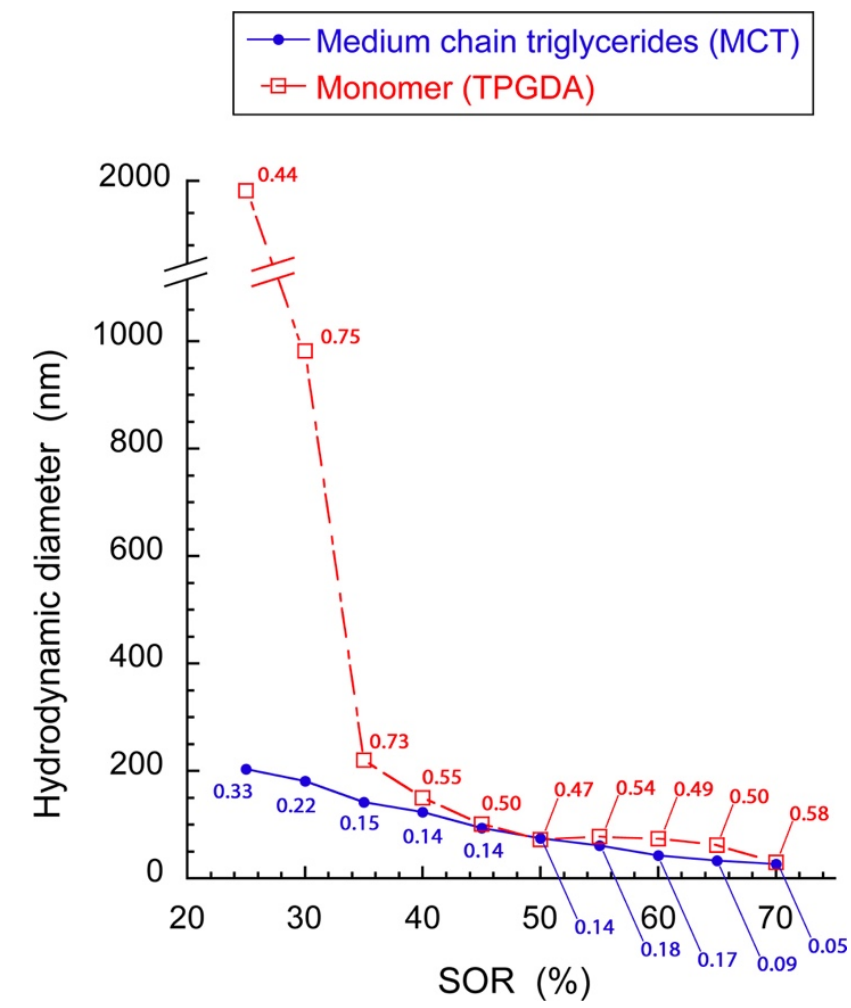

Figure 2: Impact of the surfactant amount (surfactant-to-oil weight ratio, SOR) on the size distribution of the nano-emulsions generated by spontaneous emulsification. Oil phase is either pure MCT (filled circles, Labrafac ${ }^{\circledR}$ WL), or pure monomer, TPGDA (open squares). The figures present mean hydrodynamic diameter and PDI (indicated as insets for each point) plotted against the surfactant amount (presented with the SOR parameter) $(n=3)$.

Low-energy nano-emulsification methods have been described through several methods like spontaneous emulsification or phase inversion temperature method which was recently shown to be based on similar mechanisms (Anton and Vandamme, 2009). In general, the formulation of oil-in-water emulsions is performed by suddenly mixing the oily phase containing a highHLB non-ionic surfactant fully miscible in certain conditions of temperatures with the aqueous phase. The process is driven, after the phase homogenization, by the sudden change of the surfactant solubility. Since these PEGylated surfactants were first mixed in the oily phase, when these are homogenized with the aqueous one (for which solubility is better) (Anton and Vandamme, 2011), the water spontaneously penetrated and broke up the \{oil + surfactant phase, turning the oil phase into nano-droplets dispersed in the aqueous phase according to a spinodal-like decomposition (phenomenon we described in previous reports (Anton and Vandamme, 2009). Spontaneous emulsification takes benefit of the intrinsic physicochemical properties of the surfactants, co-surfactants and excipients in the formulation, to create the 
nano-dispersion of the oily phase. As a result, the lipophilic compounds were homogeneously dispersed in the droplets, assuming that all the nano-droplets have the similar composition of MCT, monomer, co-monomer, initiator, etc. The polymerization step logically might give a population of similar droplets.

The first sets of results concerned spontaneous emulsification stage, and more particularly the outstanding emulsifying properties that can be obtained with the TPGDA monomer without oil or in combination with MCT, since these two components are extremely miscible. In the case of MCT/surfactant, as illustrated in Fig. 2 (filled circles), increasing the surfactant concentration classically resulted in gradually decreasing the droplet size, along with a rather good monodispersity i.e. PDI close to 0.15 . The global sizes exhibited a monotonous downturn from $200 \mathrm{~nm}$ for SOR around $25 \%$, up to hydrodynamic diameters below $50 \mathrm{~nm}$ for SOR higher than $60 \%$. On the other hand, when all the MCT phase was substituted for the lipophilic monomer TPGDA (Fig. 2, open squares), the trend appeared similar for SOR $\geq 40 \%$, with a size around $200 \mathrm{~nm}$ for the SOR value around $35 \%$ and decreasing below 100 or $50 \mathrm{~nm}$ for SOR $>50 \%$. However, the dispersity appeared much higher with PDI higher than 0.4, indicating that the process worked in dispersing the monomer phase, but was definitely less efficiency than with vegetable oils and created disparate size distributions. The nano-emulsions obtained with pure monomers (Fig. 2, open squares), present indeed a poor monodispersity, denoting a significant behavior as compared to pure MCT. Considering the nano-emulsification of a mixture of MCT and TPGDA as oil phase in different weight ratios for different fixed SOR (from 20 to $60 \%$ ), the oil-to-monomer weight ratio impacted the size and PDI (Fig. 3). Regarding the effect of SOR on the droplet size and PDI, the trends were close and showed a decrease in size linked to the surfactant concentration. More interesting, the increase in TPGDA concentration appeared highly beneficial for the process, impacting the droplet size, but mostly improving the monodispersity of the suspension decreasing the PDI value below 0.09-0.08 in some cases as opposed to what was observed with TPGDA alone. 


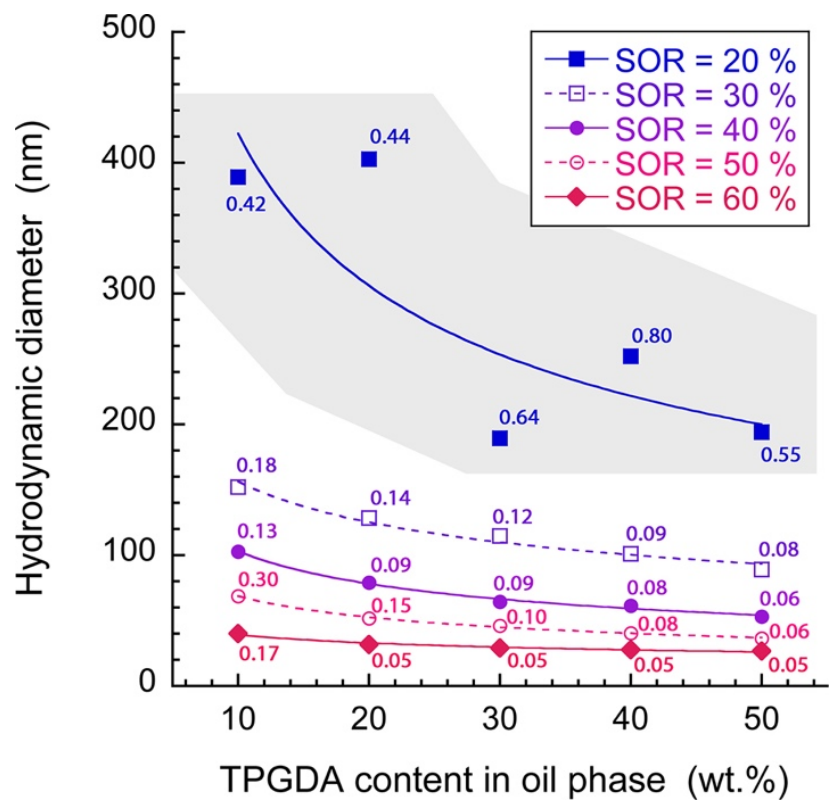

Figure 3: Nanogels formulated with the mixture of MCT and TPGDA. Hydrodynamic diameter and PDI are plotted against the TPGDA weight content, fit curves are power law, displayed on the figure to guide the eye $(\mathrm{n}=3)$. The corresponding PDI values are indicated in the figure, and the gray light region shows the formulations ( $\mathrm{SOR}=20 \%$ ) with high PDI values, indicating polydisperse samples, i.e. not fitting with the criteria of satisfactory formulations.

Mixtures of MCT and TPGDA allowed the production of smaller and more monodisperse droplets compared to pure MCT or pure TPGDA. For a SOR of $30 \%$, the size decreased from $152 \mathrm{~nm}(\mathrm{PDI}=0.18)$ down to $100 \mathrm{~nm}(\mathrm{PDI}=0.08)$ for TPGDA content in MCT of 10 to 50 wt. $\%$, respectively. In comparison, for the same SOR (30\%), the formulation with pure MCT gave $181 \mathrm{~nm}(\mathrm{PDI}=0.22)$, and even, with pure TPGDA the process did not work properly with size around $1 \mu \mathrm{m}$ and PDI $>0.7$ (i.e. DLS measurements does not have meaning). As regards the SOR $=60 \%$, the size remained constant over the change of composition of the oil phase below $50 \mathrm{~nm}$, reaching around $35 \mathrm{~nm}$ for TPGDA content at $50 \mathrm{wt} . \%$ while it was about $40 \mathrm{~nm}$ and $75 \mathrm{~nm}$ when using pure MCT and pure TPGDA, respectively. These results emphasized that the mixture of MCT and monomer TPGDA improve the emulsification process as compared with formulations with individual pure compounds. Besides the current study, these results could find some interest in the optimization of the spontaneous nanoemulsification process. In is also important to note that at a too low surfactant concentration ( $\mathrm{SOR}=20 \%$, light gray region in the figure), the spontaneous emulsification process is not working successfully, giving rise to highly polydisperse formulations, whatever the oil phase 
composition. This shows that the most important factor impacting on the nano-emulsification process, in that case, is not the oil phase composition, but rather the surfactant concentration.

\subsection{Drug-loaded nanogels}

Such nanogels have a panel of potential applications in drug encapsulation and their release can be controlled by the gel structure and density. The tunability, encapsulation of model compounds, and monitoring of its release was investigated as a function of the gel characteristics. The nanogel consist of a cross-linked network of (poly(TPGDA), poly(BA) or poly(EA)) polymer chains swollen by MCT form bi-continuous structures able to modulate the release of compounds solubilized in the oil core of the lipid nano-droplet. As a proof of concept, nanogels have been designed to encapsulate a hydrophobic drug (ketoprofen, KP) by admixing the oil phase with the drug prior to spontaneous emulsification. By varying the weight content of the cross-linker (TPGDA) and type of the monofunctional monomer (EA or BA), one expects to modulate the drug release profile by changing either the density of the cross-linked polymer network (variation of the amount of cross-linker and polymerization time), or the interaction between the drug and monofunctional monomer. The drug release profile was determined by following the concentration of the ketoprofen in the dialysate by UV spectrophotometry. Ketoprofen exhibits an absorbance peak at $260 \mathrm{~nm}$. However, it turned out that the monomers selected (TPGDA, EA and BA) also absorbed at that wavelength which may affect the determination of the drug release profile if unreacted monomers were present.

Therefore, a series of experiments without ketoprofen were conducted to establish for each monomer used its absorbance value at $240 \mathrm{~nm}$ with time following the same procedure described for the drug release. Formulation were composed of pure MCT containing different monomers (tri(propylene glycol) diacrylate or ethyl acrylate or butyl acrylate) along with hydrophilic nonionic surfactant Kolliphor ELP ${ }^{\circledR}$. The monomer was used either alone or in combination with other monomers having different ratios in oil. The results depicted in Fig. 4 showed the KP release profiles according to the composition and the presence or absence of polymers, including experiments with monomer before polymerization compared to polymer nanogel after polymerization. 


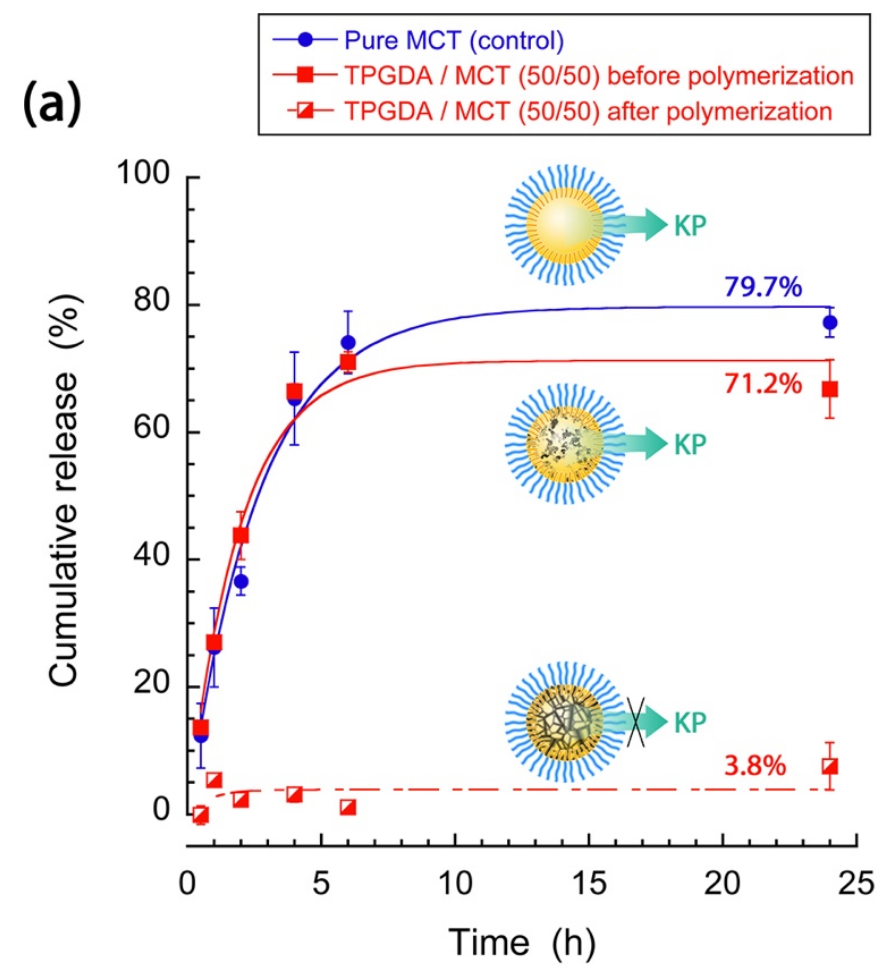

\section{(b)}

$\longrightarrow$ TPGDA / Butyl acrylate / MCT (4.5 / 42.5 / 50) after polymerization - TPGDA / Ethyl acrylate / MCT (4.5 / 42.5 / 50) after polymerization - - TPGDA / Ethyl acrylate / MCT ( 4.5 / 42.5 / 50) half-time polymerization

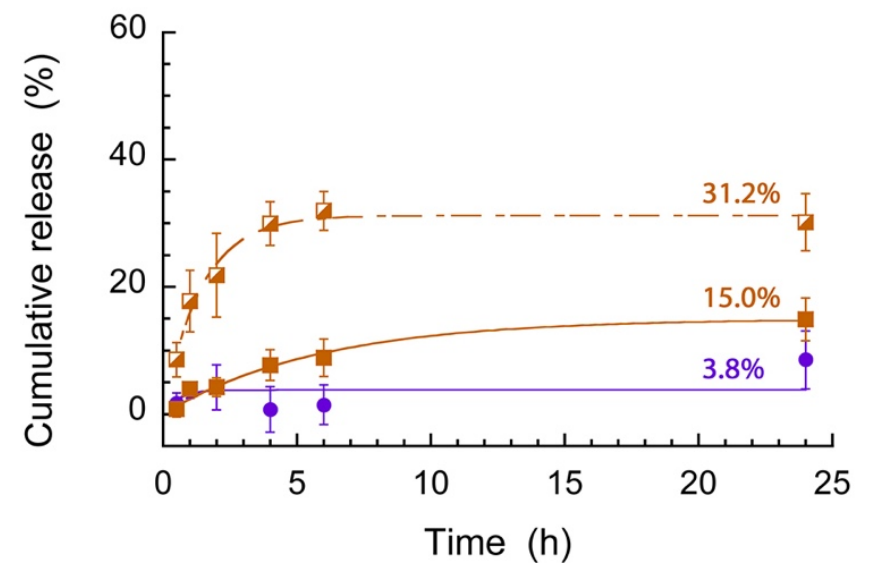

Figure 4: Release profiles in PBS of KP encapsulated in oil reservoir of nano-emulsions or nanogels, for different composition and experimental conditions $(n=3)$.

In order to ensure KP solubilization in the release medium USP phosphate buffer solution $(\mathrm{pH}=6.8)$ was chosen. In Fig 4 (a), KP was encapsulated in nano-emulsion made with pure $\mathrm{MCT}$, and a formulation composed of $50 \%$ of $\mathrm{MCT}$ and $50 \%$ of monomer, before and after polymerization. These three nano-suspensions were comparable in size and polydispersity, being $81 \mathrm{~nm}(\mathrm{PDI}=0.17), 89 \mathrm{~nm}(\mathrm{PDI}=0.19)$ and $99 \mathrm{~nm} \quad(\mathrm{PDI}=0.20)$, respectively. 
Regarding the release profiles, the curves exhibited, as expected, an exponential growth, showing a burst release and stabilizing as a plateau. The pure MCT and monomer samples actually showed a similar behavior with a significant release, giving rise to a plateau around $79 \%$ and $71 \%$, respectively. The fact that cumulative release did not reach $100 \%$ implied that a part of the drug was still encapsulated in the oil core, likely a residual solubilization of the drug, which was shared between oil and water. On the other hand, the same sample after the creation of lipogel after polymerization of TPGDA totally inhibited the drug leakage making the cumulative release around $4 \%$ after $24 \mathrm{~h}$. This is a strong evidence of the advantages brought out by the lipogel in the formulation: the polymer network indeed retained the encapsulating molecules, as expected. Interestingly, owing to the fact that monomeric residues, in theory, can interfere with the absorbance of KP, the close location of the curves (Fig. 4 (a)) of the control in pure MCT and the mixture TPGDA/MCT (50/50) before polymerization shows that their impact on the methodology is negligible, likely explained by their poor solubility in the bulk aqueous phase.

In Fig. 4 (b), investigations were extended, replacing the major part of TPGDA by $n$-butyl acrylate or ethyl acrylate (in order to open the formulations to future applications to functionalize the polymer), providing, after polymerization, droplet size around $69 \mathrm{~nm}$ $(\mathrm{PDI}=0.13)$ and $85 \mathrm{~nm}(\mathrm{PDI}=0.09)$, for ethyl acrylate and $n$-butyl acrylate, respectively. $\mathrm{KP}$ release results were comparable, for both samples after polymerization, lipogels formed in the nano-droplets induced an important drug retention -i.e. releases around $15 \%$ and $4 \%$ after $24 \mathrm{~h}$, respectively comparable to the one shown in Fig. 4 (a) with pure TPGDA. A last control was $\mathrm{KP}$ release when the polymerization was incomplete i.e. after only half of the time necessary for the full monomer conversion (i.e. $5 \mathrm{~min}$ ). The results indicated an increase of the KP leakage, reaching around $30 \%$ after $24 \mathrm{~h}$, confirming the hypothesis and previous results.

\section{Conclusion}

The general objective of this study was to create a lipogel inside the droplet core in order to decrease or inhibit the premature release of encapsulated materials. To this end, a new and facile route to synthesize lipophilic nanogels swelled with MCT was proposed. The first step was the spontaneous emulsification of a difunctional monomer (TPGDA) / MCT (Labrafac ${ }^{\circledR}$ WL) oil phase, to form nano-emulsions. Then, the second step was the photopolymerization of the nanodroplets to obtain a colloidal nanogels dispersion. Results showed that this method can be used to produce monodisperse nanogels at low surfactant amount (i.e. low SOR), with a size easily 
tunable from $c a .200 \mathrm{~nm}$ to $c a .30 \mathrm{~nm}$ according to the formulation parameters. The main advantage of nano-emulsions is their high loading capacity and we have shown in the last part of the study, that the nanogels inhibited the premature leakage of a model drug encapsulated (ketoprofen), from $c a .80 \%$ to $4 \%$ release after $24 \mathrm{~h}$, in absence or presence of the lipogel, respectively.

\section{Acknowledgments}

SD would like to acknowledge the China Scholarship Council for his Ph.D. fellowship. MB would like to acknowledge the funding support from the Higher Education Commission (Pakistan) for this research work. CS is grateful to P. Allgayer for the mechanical engineering support.

\section{References}

Anton, N., Vandamme, T.F., 2011. Nano-emulsions and Micro-emulsions: Clarifications of the Critical Differences. Pharmaceutical Research 28, 978-985. https://doi.org/10.1007/s11095-010-0309-1

Anton, N., Vandamme, T.F., 2009. The universality of low-energy nano-emulsification. International Journal of Pharmaceutics 377, 142-147. https://doi.org/10.1016/j.ijpharm.2009.05.014

Chacko, R.T., Ventura, J., Zhuang, J., Thayumanavan, S., 2012. Polymer nanogels: A versatile nanoscopic drug delivery platform. Advanced Drug Delivery Reviews 64, 836-851. https://doi.org/10.1016/j.addr.2012.02.002

García, M.C., Cuggino, J.C., 2018. Stimulus-responsive nanogels for drug delivery, in: Stimuli Responsive Polymeric Nanocarriers for Drug Delivery Applications, Volume 1. Elsevier, pp. 321-341. https://doi.org/10.1016/B978-0-08-101997-9.00016-3

Gruber, A., Işık, D., Fontanezi, B.B., Böttcher, C., Schäfer-Korting, M., Klinger, D., 2018. A versatile synthetic platform for amphiphilic nanogels with tunable hydrophobicity. Polymer Chemistry 9, 5572-5584. https://doi.org/10.1039/C8PY01123K

Hasegawa, U., Nomura, S.M., Kaul, S.C., Hirano, T., Akiyoshi, K., 2005. Nanogel-quantum dot hybrid nanoparticles for live cell imaging. Biochemical and Biophysical Research Communications 331, 917-921. https://doi.org/10.1016/j.bbrc.2005.03.228

Hayashi, C., Hasegawa, U., Saita, Y., Hemmi, H., Hayata, T., Nakashima, K., Ezura, Y., Amagasa, T., Akiyoshi, K., Noda, M., 2009. Osteoblastic bone formation is induced by using nanogel-crosslinking hydrogel as novel scaffold for bone growth factor. Journal of Cellular Physiology 220, 1-7. https://doi.org/10.1002/jcp.21760 
Khan, I.U., Serra, C.A., Anton, N., Vandamme, T., 2013. Continuous-flow encapsulation of ketoprofen in copolymer microbeads via co-axial microfluidic device: Influence of operating and material parameters on drug carrier properties. International Journal of Pharmaceutics 441, 809-817. https://doi.org/10.1016/j.ijpharm.2012.12.024

McAllister, K., Sazani, P., Adam, M., Cho, M.J., Rubinstein, M., Samulski, R.J., DeSimone, J.M., 2002. Polymeric Nanogels Produced via Inverse Microemulsion Polymerization as Potential Gene and Antisense Delivery Agents. J. Am. Chem. Soc. 124, 1519815207. https://doi.org/10.1021/ja027759q

Miller, C.A., 1988. Spontaneous Emulsification Produced by Diffusion - A Review. Colloids and Surfaces 29, 89-102. https://doi.org/10.1016/0166-6622(88)80173-2

Molina-García, L., Santos, J.L.M., Ruiz-Medina, A., Llorent-Martínez, E.J., 2013. Determination of ketoprofen based on its quenching effect in the fluorescence of quantum dots. Journal of Food and Drug Analysis 21, 426-431. https://doi.org/10.1016/j.jfda.2013.09.007

Oishi, M., Sumitani, S., Nagasaki, Y., 2007. On-Off Regulation of ${ }^{19}$ F Magnetic Resonance Signals Based on pH-Sensitive PEGylated Nanogels for Potential Tumor-Specific Smart ${ }^{19}$ F MRI Probes. Bioconjugate Chemistry 18, 1379-1382. https://doi.org/10.1021/bc7002154

O’Reilly, R.K., Hawker, C.J., Wooley, K.L., 2006. Cross-linked block copolymer micelles: functional nanostructures of great potential and versatility. Chemical Society Reviews 35, 1068. https://doi.org/10.1039/b514858h

Peng, H., Stolwijk, J.A., Sun, L.-N., Wegener, J., Wolfbeis, O.S., 2010. A Nanogel for Ratiometric Fluorescent Sensing of Intracellular pH Values. Angewandte Chemie International Edition 49, 4246-4249. https://doi.org/10.1002/anie.200906926

Qiao, Z.-Y., Zhang, R., Du, F.-S., Liang, D.-H., Li, Z.-C., 2011. Multi-responsive nanogels containing motifs of ortho ester, oligo(ethylene glycol) and disulfide linkage as carriers of hydrophobic anti-cancer drugs. Journal of Controlled Release 152, 57-66. https://doi.org/10.1016/j.jconrel.2011.02.029

Raemdonck, K., Demeester, J., De Smedt, S., 2009. Advanced nanogel engineering for drug delivery. Soft Matter 5, 707-715. https://doi.org/10.1039/B811923F

Rehman, A.U., Collot, M., Klymchenko, A.S., Akram, S., Mustafa, B., Vandamme, T., Anton, N., 2019. Spontaneous nano-emulsification with tailor-made amphiphilic polymers and related monomers. European Journal of Pharmaceutical Research 1, 27 36. https://doi.org/10.34154/2019-EJPR.01(01).pp-27-36/euraass

Ryu, J.-H., Chacko, R.T., Jiwpanich, S., Bickerton, S., Babu, R.P., Thayumanavan, S., 2010a. Self-Cross-Linked Polymer Nanogels: A Versatile Nanoscopic Drug Delivery Platform. Journal of the American Chemical Society 132, 17227-17235. https://doi.org/10.1021/ja1069932 
Ryu, J.-H., Jiwpanich, S., Chacko, R., Bickerton, S., Thayumanavan, S., 2010b. SurfaceFunctionalizable Polymer Nanogels with Facile Hydrophobic Guest Encapsulation Capabilities. Journal of the American Chemical Society 132, 8246-8247. https://doi.org/10.1021/ja102316a

Sasaki, Y., Akiyoshi, K., 2010. Nanogel engineering for new nanobiomaterials: from chaperoning engineering to biomedical applications. The Chemical Record n/a-n/a. https://doi.org/10.1002/tcr.201000008

Torchilin, V.P., 2005. Recent advances with liposomes as pharmaceutical carriers. Nature Reviews Drug Discovery 4, 145-160. https://doi.org/10.1038/nrd1632

Wang, X., Anton, N., Ashokkumar, P., Anton, H., Fam, T.K., Vandamme, T., Klymchenko, A.S., Collot, M., 2019. Optimizing the Fluorescence Properties of Nanoemulsions for Single Particle Tracking in Live Cells. ACS Applied Materials \& Interfaces 11, 13079-13090. https://doi.org/10.1021/acsami.8b22297

Yan, S., Sun, Y., Chen, A., Liu, L., Zhang, K., Li, G., Duan, Y., Yin, J., 2017. Templated fabrication of $\mathrm{pH}$-responsive poly( $\mathrm{L}$-glutamic acid) based nanogels via surfacegrafting and macromolecular crosslinking. RSC Advances 7, 14888-14901. https://doi.org/10.1039/C7RA00631D

Yu, M., Yuan, W., Li, D., Schwendeman, A., Schwendeman, S.P., 2019. Predicting drug release kinetics from nanocarriers inside dialysis bags. J. Control. Release 315, 23-30. https://doi.org/10.1016/j.jconrel.2019.09.016

Zhuang, J., Jiwpanich, S., Deepak, V.D., Thayumanavan, S., 2012. Facile Preparation of Nanogels Using Activated Ester Containing Polymers. ACS Macro Letters 1, 175179. https://doi.org/10.1021/mz200123f 